\title{
Robotically assisted perventricular closure of perimembranous ventricular septal defects: Preliminary results in Yucatan pigs
}

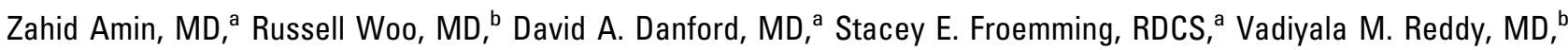
John Lof, ${ }^{\mathrm{a}}$ and David Overman, $\mathrm{MD}^{\mathrm{c}}$

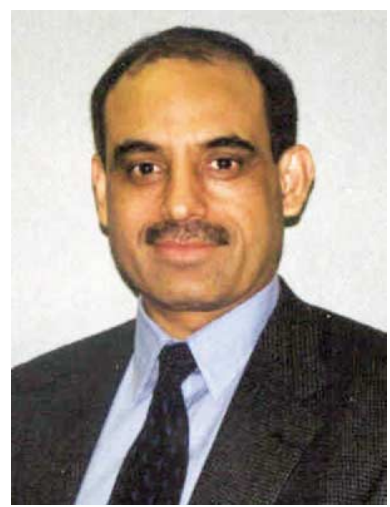

Dr Amin
From the Joint Division of Pediatric Cardi-
ology, University of Nebraska/Creighton
University, Children's Hospital of Omaha,
Omaha, Neb, 'Stanford University Medical
Center, Palo Alto, Calif, ${ }^{\text {b and Children's }}$
Health Care Minneapolis, Minneapolis, Minn. ${ }^{\text {c }}$
Supported in part by a research grant from
AGA Medical Corporation, Golden Valley,
Minn.

Read at the Eighty-fifth Annual Meeting of The American Association for Thoracic Surgery, San Francisco, Calif, April 10-13, 2005.

Received for publication Aug 15, 2005; revisions received Oct 10, 2005; accepted for publication Oct 20, 2005.

Address for reprints: Zahid Amin, MD, Children's Hospital of Omaha, 8200 Dodge St, 4th Floor Health Care Pavilion, Omaha, NE 68114 (E-mail: zamin@chsomaha.org).

J Thorac Cardiovasc Surg 2006;131:427-32

$0022-5223 / \$ 32.00$

Copyright (C) 2006 by The American Association for Thoracic Surgery

doi:10.1016/j.jtcvs.2005.10.034
Objective: Robotic systems allow surgeons to perform minimally invasive cardiac surgery in adults. Experience in the pediatric population, however, is limited. Perventricular closure of muscular ventricular septal defects has been reported in humans but requires a median sternotomy. The objective of this study was to assess the feasibility of robotically assisted closure of perimembranous ventricular septal defects by using the perventricular approach.

Methods: The procedure was attempted in 7 pigs with naturally occurring perimembranous ventricular septal defects. Echocardiography was performed to confirm the presence and assess the size of the defect. A 3-armed da Vinci system consisting of two 8-mm instrument ports and a 12-mm endoscopy port was used. A pericardiotomy was performed, and the right ventricular free wall was visualized. A spinal needle was advanced into the right ventricular cavity. By using echocardiographic guidance, a glide wire was advanced through the angiocatheter and manipulated through the defect into the left ventricle or the ascending aorta. A delivery sheath was advanced over the wire. An appropriately sized Amplatzer device was deployed through the sheath.

Results: The procedure was successful in 5 pigs. One device was removed because it was smaller than the defect and an appropriately sized device was not available. The placement failed in the second pig in the series. Four pigs were followed up for 1 to 4 months. Angiograms performed before the pigs were killed documented complete occlusion in 3 and mild-to-moderate shunt in 1 .

Conclusions: Robotically assisted perventricular closure with the Amplatzer Membranous VSD Occluder is feasible. This approach avoids the associated morbidities of cardiopulmonary bypass and median sternotomy. Further investigation and refinements are needed, however, before application of this approach in humans.

$\mathrm{P}$ erimembranous ventricular septal defect (VSD) is one of the most common congenital cardiac defects. ${ }^{1}$ Since the first successful surgical closure of VSD by Lillehei, ${ }^{2}$ open repair by using cardiopulmonary bypass (CPB) has been considered the gold standard. As survival after surgery for congenital heart defects has improved, the focus has shifted toward minimizing morbidity by using a variety of methods, among them minimally invasive surgical techniques and strategies that minimize or eliminate the use of CPB. ${ }^{3}$ The robotic Da Vinci Surgical System (Intuitive Surgical, Sunnyvale, Calif) has been successfully used for a variety of procedures in the adult population, including intracardiac repairs, but its application in the pediatric population has been limited. ${ }^{4,5}$ Furthermore, many minimally invasive or port access methods require CPB support via femoral cannulation. CPB through the femoral vessels in the pediatric population may not be feasible because the femoral vessels may be too small. Perventricular device closure of muscular 


\section{Abbreviations and Acronyms \\ $\mathrm{CPB}=$ cardiopulmonary bypass \\ VSD $=$ ventricular septal defect}

VSD has been demonstrated to be a highly successful and low-risk procedure. ${ }^{6-9}$ This technique avoids the morbidity inherent to $\mathrm{CPB}^{10}$ but requires median sternotomy. It also avoids the peripheral vascular complications associated with catheter-based device delivery and expands the availability of device closure to patients who would otherwise not be candidates for catheter-based therapy because of size restrictions or anatomic factors.

The recently developed Amplatzer Membranous VSD Occluder (AGA Medical Corporation, Golden Valley, Minn) has been successfully used to close perimembranous VSD in children by using the transcatheter technique. Perventricular deployment of the membranous device via median sternotomy has been reported in animals. With the adjunctive use of a robotic system, median sternotomy can be avoided in addition to avoiding CPB. Avoiding sternotomy and CPB should decrease the overall morbidity. The objective of this study was to evaluate the safety and feasibility of VSD closure with the da Vinci system and the perventricular technique.

\section{Materials and Methods}

All animals received humane care in compliance with the "Guide for the Care and Use of Laboratory Animals" prepared by the Institutes of Laboratory Animal Resources and published by the National Institutes of Health (publication no. 86-223, revised 1985). The study was approved by the Institutional Animal Care and Use Committee of the University of Nebraska Medical Center (Omaha, Neb).

\section{Animal Model}

Yucatan pigs, which have a naturally occurring perimembranous VSD, were used in the study. The perimembranous defect in these pigs is anatomically similar to that found in humans. The defect is located just beneath the anteroseptal commissure of the tricuspid valve. On the left ventricular side, the defect is subaortic. The rate of aneurysm formation seems to be higher in pigs than in humans, and, hence, spontaneous closure may also be higher. The course of the conduction system in relation to the defect remains unknown. Atrioventricular block after device closure has not been reported in the Yucatan model.

\section{The Device}

The Amplatzer Membranous VSD Occluder is made from 0.005inch nitinol wires. The device has been described in detail previously. ${ }^{11}$ In brief, the device is self-expanding and self-centering and can be delivered through a 7F through 9F delivery sheath. Sheath size depends on the size of the device chosen for closure. The wires are woven to form 2 connecting disks that are connected

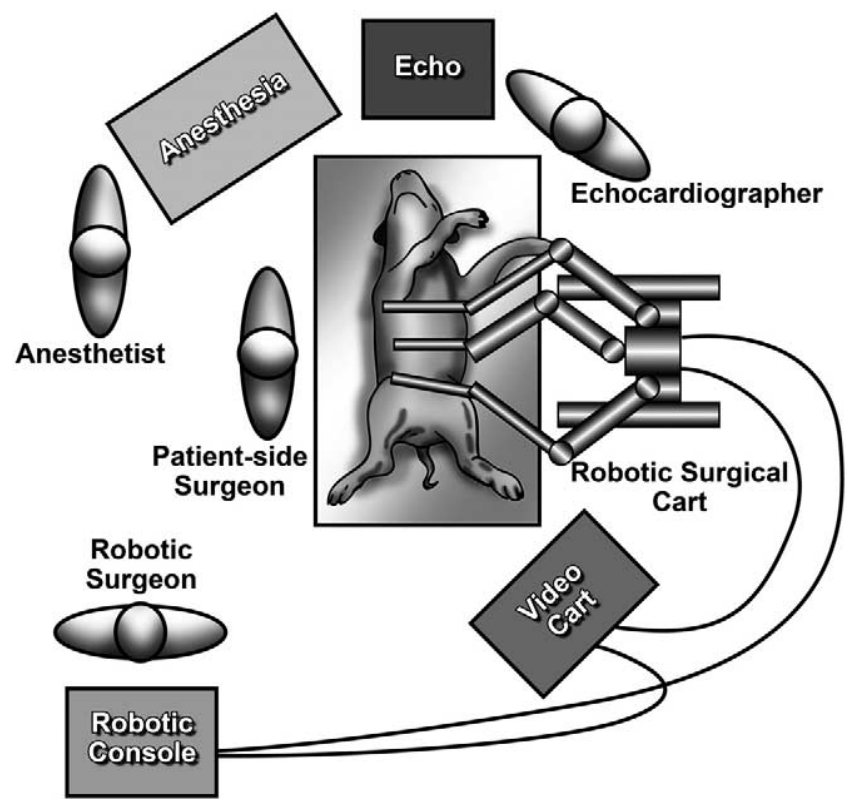

Figure 1. Layout of the operating room suite with the positions of the anesthesia, echocardiographer, anesthetist, and surgeon shown.

to each other with a connecting waist. The disks are $1.5 \mathrm{~mm}$ apart. The waist of the device corresponds to the size of the defect. The left disk is eccentric in design; the portion of the device that is to face the aortic valve is flat instead of round. The flat-edge design is meant to avoid impingement of the aortic cusps. The delivery sheath has a coaxial system and is designed to lock the device so that at the time of deployment, the flat edge of the device will face the aortic valve. The edge of the device that is to face the left ventricle apex is $5.5 \mathrm{~mm}$ larger than the waist. The right-sided disk is $2 \mathrm{~mm}$ larger than the waist in its entire circumference. A platinum marker is present on the left disk; this marker helps the operator maintain an optimal device orientation at the time of the delivery. The marker is placed on the side of the disk that is to face the left ventricular apex. On fluoroscopy, the marker should be at 1800 hours in the left anterior oblique view with cranial tilt.

\section{Echocardiogram}

Transesophageal echocardiographic images are not obtainable in Yucatan pigs; therefore, intraoperative transthoracic echocardiography was performed. With the animal in the left decubitus position, transthoracic echocardiography was performed by using a commercially available 2-dimensional echocardiographic imaging system with color flow mapping and pulsed and continuous-wave Doppler capabilities. Imaging frequencies ranged from 3 to 8 MHz. Most examinations required the lower frequencies for optimal ultrasound penetration. Modified apical and long-axis 2dimensional echocardiographic views were most commonly obtained from the anterior left axillary line transducer position. Wide variations in cardiac position and orientation within the thorax were encountered from animal to animal, so a search for the point on the chest wall that yielded optimal imaging was required in 


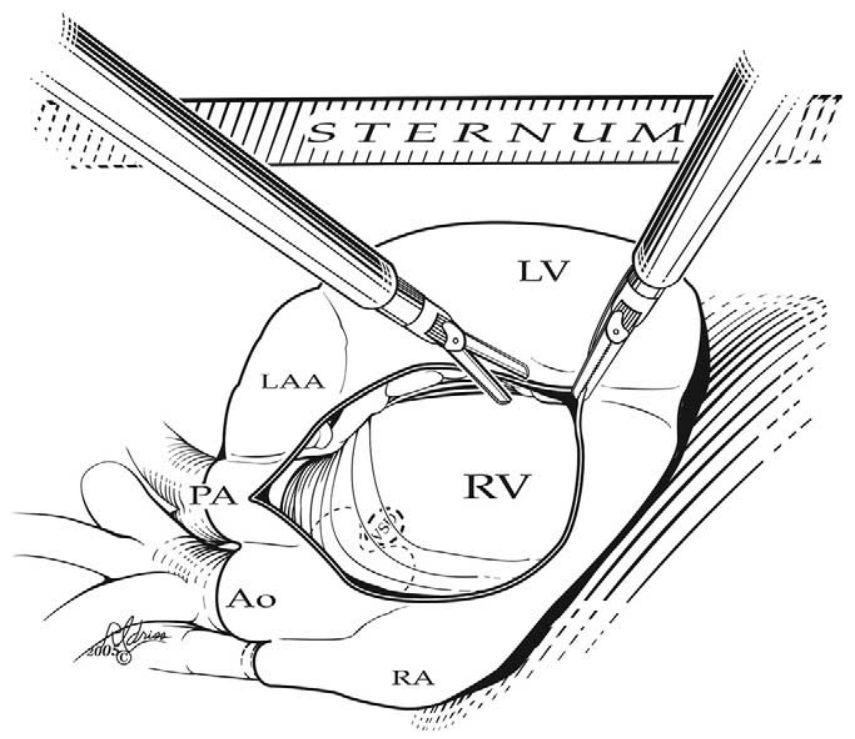

Figure 2. Robotically assisted pericardiotomy. $R V$, Right ventricle; $R A$, right atrium; $A o$, aorta; $L V$, left ventricle; $P A$, pulmonary artery; $L A A$, left atrial appendage.

each case. Color flow mapping aided in the rapid identification of the VSD. Once the defect was located, its proximity to the aortic and tricuspid valves was evaluated. A search for preintervention pathologic flow disturbances across the cardiac valves was undertaken by using color flow mapping. Digital measurements of the VSD diameter were obtained from freeze-frame images of the 2-dimensional image and from the breadth of the color Doppler shunt signal on the flow map. Positioning of the device delivery apparatus, the delivery of the device, and its release were monitored echocardiographically. The final position of the device relative to the defect and neighboring cardiac valves was evaluated on the 2-dimensional images in multiple planes, and any new or persistent pathologic flow disturbances upon device delivery and release were noted.

\section{The Procedure}

The general outline of the operating room suite is depicted in Figure 1. All procedures were performed under single-lumen general endotracheal tube anesthesia with standard anesthesia monitoring. A certified veterinary anesthetist was present and delivered the anesthesia at all times. The animals were positioned in a modified $\left(10^{\circ}\right)$ left lateral decubitus position on the operating table. The forelimbs were retracted cephalad and to the left by using soft restraints. A soft roll was placed under the scapula to widen the intercostal spaces. A 3-armed da Vinci Surgical System was then positioned at the left side of the table, with the surgeon at the right side of the table. The head of the table and the vision cart were positioned at the foot of the table. The robotic surgeon was positioned at the master surgeon's console away from the operative field (Figure 1). The components and operation of the da Vinci Surgical System have been described previously. ${ }^{5}$ In brief, 3 ports were used for all operations: an 8-mm left da Vinci instru-
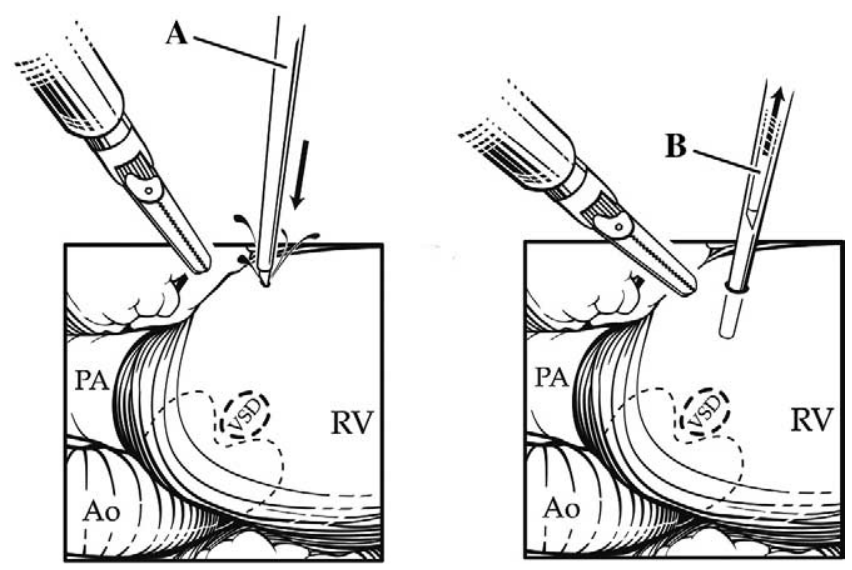

Figure 3. A, The spinal needle introduced through the chest wall. $B$, The stylet is removed. Ao, Aorta; VSD, ventral septal defect; RV, right ventricle; $P A$, pulmonary artery.

ment port 3 to $4 \mathrm{~cm}$ lateral to the right sternal border in the most cephalad intercostal space possible, a 12-mm endoscopic port placed 6 to $8 \mathrm{~cm}$ caudal and 1 to $2 \mathrm{~cm}$ lateral to the left instrument port, and one $8-\mathrm{mm}$ right da Vinci instrument port placed 6 to $8 \mathrm{~cm}$ caudal to the endoscopic port and 3 to $4 \mathrm{~cm}$ lateral to the right sternal border. A $12-\mathrm{mm}, 30^{\circ}$ stereo endoscope in the angled-down position provided the best visualization. A thoracotomy was not performed. Carbon dioxide insufflation was not necessary.

A pericardiotomy was performed anterior to the phrenic nerve by using robotic hook electrocautery and forceps and was extended sufficiently to allow wide visualization of the right ventricular free wall and the outflow tract (Figure 2). ${ }^{2}$ The delivery sheath was inserted percutaneously into the right ventricular free wall. Once the delivery sheath was successfully positioned (see below), a double armed 4-0 monofilament purse-string suture was robotically placed around it and held under tension to ensure hemostasis at the right ventricular puncture site during device deployment. After successful closure and removal of the sheath, the purse string was tied securely with the help of robotic instruments.

The technique of perventricular closure of VSD with the Amplatzer Membranous VSD Occluder in Yucatan pigs has been described previously. ${ }^{11}$ All procedures were performed with the help of transthoracic echocardiography guidance. The VSD was measured in 2 views. A 16-gauge spinal needle (Cook Inc, Bloomington, Ind) was advanced through the fifth intercostal space (Figure 3) and clearly visualized endoscopically (Figure 3, $a$ ). The right ventricular free wall was punctured at the desired location, and the needle was visualized in the right ventricular cavity by echocardiography (Figure 3,b). The stylet was removed, and a glide wire (Meditech, Boston Scientific Corporation, Natick, Mass) was advanced through the needle into the right ventricular cavity (Figure 4) and then manipulated across the VSD into the left ventricle or the ascending aorta (Figure 4, A). The spinal needle was removed, and a 9F dilator was advanced over the wire to predilate the entry before advancing the delivery sheath. A delivery sheath was advanced over the wire into the left ventricular cavity (Figure $4, B$ ), and its position was confirmed by echocar- 

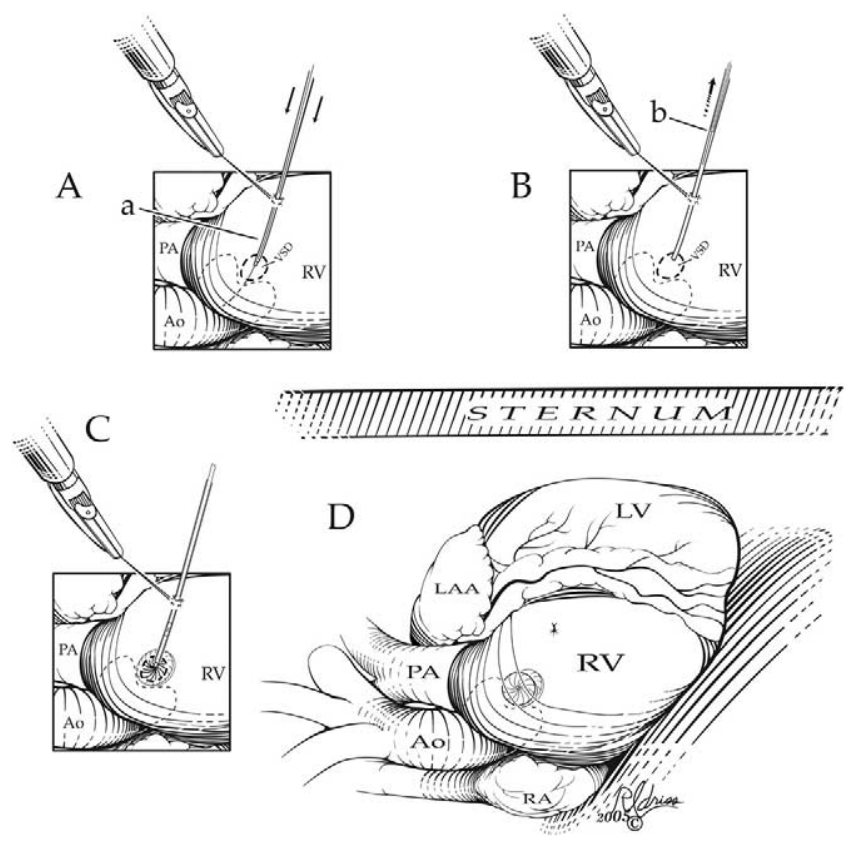

Figure 4. A, Glide wire inserted through the needle and VSD and into the aorta. B, The delivery sheath introduced over the wire. C, The device introduced through the delivery sheath, attached to the delivery cable. $D$, The device has been released. Ao, Aorta; VSD, ventral septal defect; $R V$, right ventricle; $P A$, pulmonary artery; $L V$, left ventricle; $L A A$, left atrial appendage.

diography. The dilator and the wire were removed, and the sheath was allowed to back-bleed to remove any entrapped air. The sheath was gently flushed with saline. An appropriately sized Amplatzer Membranous VSD Occluder was immersed in saline and screwed onto the delivery cable. The device was loaded with the help of a loader and advanced through the delivery sheath. Once the device, which was visible while within the sheath, was seen to cross the VSD by echocardiography, the sheath was withdrawn, and the left disk was deployed. If the orientation of the left disk seemed suboptimal, then the sheath was rotated until optimal orientation was achieved. The device and the sheath were pulled back together to approximate the left disk to the ventricular septum. The right disk was then deployed (Figure 4, C). The position of the device was again confirmed, and the integrity of the aortic and the tricuspid valves was evaluated. The device was released by anticlockwise rotation of the delivery cable (Figure 4,C). After removal of the sheath and tying of the purse-string suture, hemostasis was verified, all ports were removed, and all port sites were closed with absorbable sutures. All animals were extubated in the operating room and recovered under monitored care for several hours. Once stable, the animals were returned to the animal facility for the remainder of their postoperative course.

During follow-up, the animals were monitored for signs of wound infection, pneumonia, and other postoperative complications. At the conclusion of the follow-up, cardiac catheterization with left ventricular and ascending aortic angiography was per- formed to evaluate VSD closure and aortic valve function. The animals were then killed for autopsy evaluation.

\section{Results}

VSD closure was attempted in 7 pigs (age, 245-384 days; weight, $35-74 \mathrm{~kg}$ ). The mean follow-up for all pigs with successful closure was 31 days (range, 1-100 days).

The procedure was successful in 6 pigs, and device placement was successful in 5. The first pig was killed at the conclusion of the procedure to directly examine the deployed device and its position. The remaining 4 pigs were observed for a minimum of 1 month before they were killed. Left ventricular angiography revealed complete closure of the VSD in 3 and mild to moderate shunt in 1 . This pig had aneurysm formation with multiple holes on the right ventricular side. Aortic angiography revealed no evidence of aortic insufficiency in any pig. No evidence of heart block or arrhythmia was seen with continuous electrocardiographic monitoring during cardiac catheterization.

In 2 pigs, the procedure was unsuccessful. The wire was not able to be passed through the defect in 1 pig, and in the other pig, the device was not released because an appropriately sized device was not available.

Examination of the heart of the pig that was killed on the day of the procedure revealed proper device orientation and position without encroachment of the aortic or the tricuspid valve. Autopsy of the other pigs also showed acceptable position and orientation of the device in each case.

\section{Discussion}

Perventricular device closure of VSD offers many advantages over other forms of therapy, including avoidance of CPB and applicability for patients who are too small for catheter-based therapy. Until now, the perventricular method has been used with a median sternotomy. Robotic systems have been used to avoid minimally invasive cardiac surgery, thus avoiding the morbidity of a full sternotomy. Because of the freedom of movement of their tips, they have a significant technical advantage over traditional endoscopic instrumentation. However, endoscopic instruments are cheaper and could potentially be used for the same purpose.

This is the first report of successful perventricular VSD closure by using robotic assistance. The blending of these 2 technical elements creates the ability to correct a common intracardiac defect without CPB or median sternotomy. This latter point is particularly important for the VSD patient, who most often requires therapy early in infancy. It is important to note that to be able to close defects in the younger population, we will need smaller ports, which may become available in the future. Further miniaturization of ports and instruments will facilitate the application of this technology even in neonates.

Since its introduction in 1997, perventricular device closure has been successfully applied in more than 40 patients 
with muscular VSD and 3 patients with perimembranous VSD (unpublished data). For muscular VSD, the procedure requires a complete sternotomy or ministernotomy. The defects are closed under transesophageal echocardiographic guidance. The delivery of the device and its placement are relatively easy because echocardiographic images are excellent. In pigs, the echocardiography pictures were not optimal because of several limitations. First, transesophageal echocardiography cannot be performed. Second, transthoracic pictures are suboptimal in pigs because the heart is midline in position. Third, the significant thickness of the chest wall limits ultrasound penetration. Fourth, the physical space available to perform echocardiography was very limited because of the robotic ports and the left decubitus position of the pig at the time of the procedure. We believe that these issues will be resolved when the procedure is attempted in humans, especially because transesophageal echocardiography will be performed with optimal echocardiographic images.

Introduction of the needle into the right ventricle will be easier in humans because of the standard familiarity of the heart position and orientation in human beings. Despite all the challenges, with refinements, we believe that the VSDs will be closed successfully in humans with robotic assistance. When the instrument ports and endoscopes become smaller, the technique can be used in humans. With the currently available size of the ports, it is not possible to use this technique in small children.

It is predicted that in the near future, robotic assistance will be an attractive option for patients and families because it will help in improving the cosmetic appearance of the patients, will decrease morbidity and hospital stay, and will be a favorable alternative to percutaneous closure in small children. The advantages of perventricular closure include the following: no CPB or its associated complications, no fluoroscopy-related or cardiac catheterization-related complications, and the ability to close defects that are otherwise difficult to close when the patient is on CPB.

In summary, this study demonstrates that robotically assisted perventricular closure of VSDs is feasible. It has the potential of minimizing morbidity associated with VSD closure, improving cosmesis, and expanding the patient population to whom device closure of defects may be offered.

The authors are grateful to Dr Emile Bacha for his valuable suggestions in reviewing the manuscript.

\section{References}

1. Kidd L, Driscoll DJ, Gersony WM, et al. Second natural history study of congenital heart defects: results of treatment of patients with ventricular septal defects. Circulation. 1993;87(suppl 1):138-51.

2. Lillehei CW. Controlled cross circulation for direct vision intracardiac surgery; correction of ventricular septal defects, atrioventricularis communis, and tetralogy of Fallot. Postgrad Med. 1955;17:388-96.
3. Lin PJ, Chang C, Chu J, et al. Minimally invasive cardiac surgical techniques in the closure of ventricular septal defect: an alternate approach. Ann Thorac Surg. 1998;65:165-70.

4. Lanfranco AR, Castellanos AE, Desai JP, Meyers WC. Robotic surgery: a current perspective. Ann Surg. 2004;239:14-21.

5. Luebbe BN, Woo R, Wolf SA, Irish MS. Robotically assisted minimally invasive surgery in a pediatric population: initial experience, technical considerations, and description of the da Vinci® Surgical System. Pediatr Endosurg Innovative Techniques. 2003;7:385-402.

6. Amin Z, Gu X, Berry JM, Titus JL, Gidding SS, Rocchini AP. Perventricular closure of ventricular septal defects without cardiopulmonary bypass. Ann Thorac Surg. 1999;68:149-54.

7. Amin Z, Berry JM, Rocchinni AR, Bass JL. Intraoperative closure of muscular ventricular septal defect in a canine model and application of the technique in a baby. J Thorac Cardiovasc Surg. 1998;115:1374-76.

8. Amin Z, Berry JM, Danford DA, Foker J, Duncan KF, Bass JL. Intraoperative closure of muscular ventricular septal defects without cardiopulmonary bypass: preliminary results of the perventricular approach. Circulation. 2001;104(suppl):17:II-710.

9. Bacha EA, Cao QL, Starr JP, Waight D, Ebeid MR, Hijazi ZM. Perventricular device closure of muscular ventricular septal defects on the beating heart: technique and results. J Thorac Cardiovasc Surg. 2003;126:1718-23.

10. Rose EA. Off-pump cardiopulmonary bypass surgery. $N$ Engl J Med. 2003;348:379-80.

11. Amin Z, Danford DA, Lof J, Duncan KF, Froemming SF. Intraoperative device closure of perimembranous ventricular septal defects without cardiopulmonary bypass: preliminary results of the perventricular technique. J Thorac Cardiovasc Surg. 2004;127:234-41.

\section{Discussion}

Dr Richard G. Ohye (Ann Arbor, Mich). Before I start, I should preface some of my comments with saying I do believe that this is important technology, but I will take a little bit of the devil's advocate role here. I have 2 somewhat related questions.

Dr Amin, in your background slides and also in your conclusions, you cite open sternotomy with $\mathrm{CPB}$ as one of the comparisons, as well as cardiac catheterization-based closure, and you cite that some of the disadvantages are the need for CPB as well as the full sternotomy, which I think is probably not the proper comparison. I have done these, and they can be done through a little mini subxiphoid incision without CPB.

In the comparison with cardiac catheterization-based intervention, you note that it is difficult to do this in less than $8 \mathrm{~kg}$ in the catheterization laboratory, and certainly most literature would say that less than $15 \mathrm{~kg}$ is the limit for da Vinci approaches. I have done them as low as $10 \mathrm{~kg}$, but it does become challenging at that size. You also note that concomitant lesions may be a problem in the catheterization laboratory, but again, that is the same for da Vinci closure; you can't do concomitant lesions either. So I think the more appropriate comparison would be an off-bypass with a small subxiphoid approach. I would like to know what you think the advantages are of using the robotic approach for that.

A related question, in a more global sense: How do you convince the chief financial officer of your hospital to buy this $\$ 1$ million plus instrument with over a $\$ 100,000$ a year cost for disposables and maintenance to do operations that are actually going to cost the hospital money? As you are well aware, the most lucrative patient for a hospital is the patient who has an operation, goes to the intensive care unit for a day or two, goes to the floor for another 2 or 3 days, and then goes home. So you are going to ask him to buy a very expensive piece of equipment to actually cost them money in patient care as well. Although I think this is 
important technology, the 2 questions I have are as follows: First, what are the advantages of a da Vinci closure versus a small subxiphoid approach? Second, in a more global sense, what do you think the importance and the advantages of robotic approaches are?

I would like to congratulate Dr Amin on a very interesting paper and an attempt to advance technology that I think will be very important in the future.

Dr Amin. I think there is definite advantage in using a robotic system with the perventricular approach. I agree with you that the weight limit for da Vinci system is $15 \mathrm{~kg}$ and hence does not appear feasible at the current time. In the future, however, as we progress further, this technique will only get better. I believe that the subxiphoid approach is a feasible technique for certain procedures, but with robotic assistance, our access to the heart will improve, and the chance of repairing defects will improve in the future, as this technology improves.

Second, I agree with you, it is costly, but then everything is costly nowadays. We use the robots all the time in the research laboratory to improve our patient care, and I think, given that reason, and the more we refine, it will ultimately pay for its cost. We cannot be satisfied with our improved results so much that we stop exploring techniques that may advance medical science.

Dr Ludwig K. von Segesser (Lausanne, Switzerland). We have used Amplatzer devices for sealing off transventricular access orifices in valve stent implantations, and I wanted to ask you what blood loss you observed for robotic closure in your application.

Dr Amin. During this experiment in the first 3 pigs, when our procedure time was longer and we actually had to puncture the right ventricular free wall more than once to find the appropriate spot through which we could advance the wire, I would say the blood loss was at least 30 to $50 \mathrm{~mL}$. In the last 4 pigs where we performed this procedure, the blood loss was less than $10 \mathrm{~mL}$.

Dr Pedro del Nido (Boston, Mass). Dr Amin, I would like to echo some of your comments. If we use cost as a way to limit our exploration of new technology, we are going to do very little that is new. The question I have relates to imaging. You used transesophageal echo, which in the pig is very challenging. Did you do anything special to actually be able to view the structures well? In an ideal world, what would you use for your imaging technology if you could use multimodality imaging?

Dr Amin. Thank you, Dr del Nido. We used transthoracic echo for this protocol because, as you said, transesophageal echocardiography cannot be performed in pigs. The perventricular closure in humans is performed under transesophageal or epicardial echocardiographic guidance. Ideally, I would be very tempted to use 3-dimensional echocardiography, if that was available, and we are planning to use it in the future experiments.

Dr Emile Bacha (Chicago, Ill). Congratulations, Dr Amin. I think you should continue to pursue this line of work. My question relates to what your goal is. As Dr Ohye was saying, the subxiphoid approach does afford very good exposure to the heart, but you are not limited to the subxiphoid approach. You can also do a small thoracic incision that would give you access to different planes to the ventricle, and I do think that the goal here is a transthoracic puncture through the chest wall into the ventricle and then delivery of the device. In that sense, the robot would be just a step on the way and eventually would probably be discarded in that technique.

Do you see any devices coming up whereby you could control the ventricular puncture from a remote approach, such as a transthoracic approach, without placing a suture on the ventricle?

Dr Amin. I am very much familiar and know that you have done a significant number of cases with a perventricular approach. Most of the time when we puncture the right ventricular free wall, the bleeding is through the angiocatheter. Once that is stopped there is usually no bleeding. At the current time, I am not aware of any device or technique that will allow us to perform this procedure without placing a suture on the right ventricular free wall. I do believe it will be doable in the future.

Regarding the advantage of robots, as I was trying to explain to Dr Ohye, I think it will have an advantage, because I think the perimembranous VSDs are more difficult to cross, and even if you want to do the procedure through a subxiphoid approach, it may be more difficult. Having said that, yes, you are right, you can do a small thoracotomy on the right side and use that approach, but I believe that we should keep experimenting and see what will be the best way to do so in the future.

\section{Targeted}

The Journal of Thoracic and Cardiovascular Surgery gives you two tables of contents.

The condensed table of contents tells you at a glance what topics and authors are presented each month. The expanded table of contents gives you a brief abstract of each article. You select only those articles of most interest to you for further reading. 